\title{
Effect of early Trimetazidine on infarct size in ST- segment-elevation myocardial infarction patients undergoing primary percutaneous coronary intervention
}

\section{GENG QIAN ( $\sim$ qiangeng9396@263.net)}

Chinese PLA General Hospital https://orcid.org/0000-0002-3238-5860

\section{Ying Zhang}

Chinese PLA General Hospital

\section{Xin A}

Chinese PLA General Hospital

\section{Xiaosi Jiang}

Chinese PLA General Hospital

\section{Zichao Jiang}

Chinese PLA General Hospital

Tao Li

Chinese PLA General Hospital

Wei Dong

Chinese PLA General Hospital

Jun Guo

Chinese PLA General Hospital

\section{Yundai Chen}

Chinese PLA General Hospital

\section{Research Article}

Keywords: Myocardial infarction, Trimetazidine, Percutaneous coronary intervention, cardiac magnetic resonance, Infarction size

Posted Date: March 16th, 2021

DOl: https://doi.org/10.21203/rs.3.rs-285838/v1

License: (9) (1) This work is licensed under a Creative Commons Attribution 4.0 International License. Read Full License 


\section{Abstract}

Purpose: Trimetazidine, a metabolic agent with anti-ischemic effects, was reported to reduce reperfusion injury in animal models. In this randomized double-blind placebo-controlled trial, we investigated the effects of trimetazidine on reducing infarction size in patients undergoing revascularization for STsegment elevation myocardial infarction (STEMI).

Methods: Patients with STEMI randomly received trimetazidine $(n=87)$ or placebo $(n=86)$ in a doubleblind manner before primary percutaneous coronary intervention (PCI), and study treatment was maintained for 12 months after the procedure. The primary endpoint was infarction size measured by cardiac magnetic resonance (CMR) after primary $\mathrm{PCl}$.

Results: The clinical characteristics of patients ( $90 \%$ male, mean age $57 \pm 12$ years) in both groups were well-matched on the baseline. Compared with patients in control group, the percentage and weight of infarction size of patients in trimetazidine group were both significantly lower $(22.1 \pm 11.8 \%$ [ $=74]$ vs. $26.9 \pm 11.9 \%[n=74], p=0.010 ; 28 \pm 18 g[n=74]$ vs. $35 \pm 19 g[n=74], p=0.022)$, the myocardial microvascular obstruction (MVO) rate measured by CMR was lower in trimetazidine group (29.7\% [22/7] vs. 52.7\% [39/74], $p=0.007$ ), while myocardial salvage index (MSI) was significantly higher in trimetazidine group $(48 \pm 20 \%$ vs. $39 \pm 27 \%, p=0.008)$. The incidence of readmission due to aggravated heart failure in trimetazidine group was lower than that in the control group without significance ( $8.0 \%$ vs $14.0 \%$, $p=0.234)$.

Conclusions: Our study provides suggests that trimetazidine initiated prior to primary $\mathrm{PCl}$, improves myocardial infarct size, MVO and MSI, possibly by reducing reperfusion injury.

\section{Introduction}

Reperfusion injury, which occurs in ST-segment elevation myocardial infarction (STEMI) after primary percutaneous coronary intervention (PCl), could further increase myocardial infarct size and lead to poor prognosis. Reperfusion injury has been increasingly concerned on its underlying mechanism and potential treatment. There is growing evidence that trimetazidine (TMZ) applied in patients with acute myocardial ischemia is beneficial in cardioprotection and reducing major cardiac events[1-2]. A previous retrospective study showed that administration of TMZ could improve clinical outcomes in patients with acute myocardial infarction (AMI) by significantly reducing all-cause mortality in the following 12 months [3]. TMZ could protect cardiomyocytes in hypoxia-ischemia via modulating energy metabolism. Experimental studies performed in animal models of cardiac ischemia/reperfusion injury have revealed that TMZ could reduce myocardial ischemia/reperfusion injury by various mechanisms, mostly due to it facilitating energy from $\beta$-oxidation of fatty acid to more efficient glucose oxidation and antioxidant property [4-7]. However, there still lacks research to provide evidence of effects of TMZ on infarct size in STEMI patients who underwent primary PCI [8]. Cardiac magnetic resonance (CMR) has emerged as the imaging modality for assessing the cardioprotective efficacy in patients with AMI. Late gadolinium 
enhancement (LGE) on CMR provides a reliable method to evaluate the infarction size. CMR could also provide the myocardial salvage index (MSI) which demonstrates efficacy of cardioprotective interventions in situations without reduction in absolute infarction size [9-10]. In this study, CMR was used to evaluate the effect of early TMZ administration on infarction size and MSI in patients who underwent primary PCI.

\section{Material And Methods}

\subsection{Compliance with Ethical Standards}

From October 2016 to October 2019, we conducted a prospective, single-center, randomized, double-blind study in the Chinese PLA General Hospital in Beijing.

The study complied with the Declaration of Helsinki, which was approved by the Beijing Ethics Association and the Ethics Committee of the Chinese PLA General Hospital. Informed consent was obtained from all individual participants included in the study. The trial was registered on ClinicalTrials.gov (registration number: NCT02826616). There is no potential conflicts of interest.

\subsection{Study population}

The study population included patients diagnosed with STEMI for the first time and underwent primary $\mathrm{PCl}$ within 12 hours of the onset of chest pain. Diagnosis of STEMI was based on the concurrence of symptoms consistent with STEMI for $>30$ minutes; and ST-segment elevation $\geq 1 \mathrm{~mm}$ in at least 2 or more inferior leads or $\geq 2 \mathrm{~mm}$ in at least 2 or more contiguous precordial leads. Patients were excluded if they met any one of the bellow conditions: already treated with TMZ, history of myocardial infarction, mechanical complications, previous coronary artery bypass grafting (CABG) or $\mathrm{PCl}$, contraindications of CMR, liver or kidney failure, malignant tumor.

\subsection{PCI Procedures}

Eligible patients were randomly assigned to receive $\mathrm{TMZ}$ or placebo before primary $\mathrm{PCl}$. Primary $\mathrm{PCl}$ was performed by 2 operators using standard techniques. Patients took $300 \mathrm{mg}$ aspirin in the emergency department, followed by $100 \mathrm{mg}$ aspirin per day orally thereafter. A loading dose of $180 \mathrm{mg}$ ticagrelor was administered before catheterization, followed by $90 \mathrm{mg}$ ticagrelor twice daily for the next 12 months. Drug-eluting stents were the stents of choice. All patients were given enoxaparin treatment every 12 hours after primary $\mathrm{PCl}$ for 7 days. Blood samples were taken for troponin T testing before and at $6,12,24,48$ hours after $\mathrm{PCl}$. The application of thrombectomy and intro-aortic balloon pump (IABP) was at the discretion of the operators. The $\mathrm{PCl}$ data analysts were kept blind from grouping.

\subsection{Experimental Treatment Protocol}

All patients were informed of the potential benefits and risks of the trial before they signed written informed consents and enrolled in the study. 
Patients were randomly divided into placebo and TMZ groups by using a computer-generated sequence at a ratio of 1:1. Patients were treated with a dose of $60 \mathrm{mg} \mathrm{TMZ} \mathrm{(Servier} \mathrm{companies)} \mathrm{or} \mathrm{placebo} \mathrm{orally}$ prior to reperfusion by primary $\mathrm{PCl}$ and followed by $20 \mathrm{mg} \mathrm{TMZ}$ or placebo three times a day for 12 months. Other medications including aspirin, ticagrelor, statins, $\beta$-blockers and ACEI/ARB were given in accordance with the European Cardiology Society STEMI guideline [11].

\subsection{Primary endpoint and clinical Follow-Up}

The primary endpoint was the infarction size measured by CMR at 7 days after primary PCl. The secondary endpoint was MSI measured by CMR at 7 days after primary $\mathrm{PCl}$ and main adverse cardiac events (MACEs) in following 12 months. We followed up these patients by routine clinical visits and recorded any MACEs until 12 months after the $\mathrm{PCl}$.

Follow-up information was obtained at the outpatient clinic.

Good clinical practice training was required for all personnel involved in the trial. MACEs were defined as stroke, repeat revascularization, and readmission due to acute heart failure, nonfatal myocardial infarction and all-cause death. All end-point events were adjudicated by an independent clinical events committee based on medical record. All were blinded to treatment group.

\subsection{Cardiovascular magnetic resonance acquisition and analysis}

The scan was performed at 7 days after primary $\mathrm{PCl}$ so as to assess the final infarction size, microvascular obstruction (MVO), area at risk (AAR) and MSI.

All participants took a CMR examination on a 1.5T MR Scanner (Achieva; Philips Medical Systems, Best, the Netherlands), using the 32-channel phased-array body coil.

All CMR images were acquired during breath-hold and with ECG triggering. Retrospective ECG gated cine CMR imaging was performed with steady-state free precession (SSFP) sequences using the standard protocol covering short axis and long axis in the 2- and 4- chamber views. The SSFP cine images were acquired continuously from the mitral annulus to the apical level without gaps on the short-axis. Based on the retrospective triggering, 25-30 cardiac phases covering systole and diastole within a cardiac cycle were reconstructed. Infarction size was acquired by the CMR LGE method which acquired the images after $15 \mathrm{~min}$ injection of gadolinium $(0.1 \mathrm{mmol} / \mathrm{kg}$ at $3 \mathrm{ml} / \mathrm{s})$. Inversion-recovery CMR LGE images were obtained at end-diastolic on short axis and the inversion time was manually adjusted to null the signal from remote myocardium. Myocardial edema-sensitive black-blood T2-weighted short tau inversionrecovery (STIR) sequence was performed using a fat-saturation triple inversion-recovery sequence. A full stack of LGE and T2 were acquired with about 10 slices of left ventricle (LV) chamber from base to apex, $8 \mathrm{~mm}$ apart. Each slice of LGE and T2 owed the same parametric location which was available for the precise analysis afterward.

The CMR data were analyzed by 2 CMR readers using the freely available validated cardiovascular image analysis software CVI42 5.11.2 (Circle Cardiovascular Imaging Inc, Calgary, Canada, version 5.10.1). Cine 
images on short-axis were used to analyze LV systolic function. The LV function analysis included all slices from end-diastole to end-systole. Left ventricular ejection fraction (LVEF) was calculated by manually tracing the endocardial borders in all phases on an ECG-triggered balanced steady-state-free procession cine sequence by applying multiple slices in the short-axis image covering the entire LV. Specifically, after manual tracing of the epicardial and endocardial borders, the areas of STEMI were quantified in LGE images detected by semi-automated software, by the method of mean + 5SD Ref ROI with manual correction (Fig. 1A-B). We removed the artifact in the remote myocardium which would affect the infarction size calculation in the LGE image. Infarction size was expressed both in grams and in the percentage of the total LV mass. The myocardial AAR was assessed as edema on the CMR scan using T2-weighted STIR sequence (Fig. 1C-D). AAR was defined as the long signal area on T2-weighted images, and was semi-automated recognized by mean +2 SD Ref ROI. We removed the artifact in the remote non-infarcted myocardium in the T2 STIR image with the manual correction to analyze AAR, and the area of MVO should be manually included in AAR. The MSI was calculated as follows: (AAR, $g$ infarction size, g)/ AAR, g. MVO was identified in LGE images as a subendocardial region with lower enhancement than the surrounding area which was semi-automated discerned via the dark areas inside the infarcted myocardium tissue with manual correction. All the data were validated by inter-and intraobserver analysis of reproducibility method.

\subsection{Statistical analysis}

Continuous variables were presented as mean \pm SD and compared by the $t$-test for independent samples. Non-normally distribution variables were presented as a median and interquartile range, and compared by the Wilcoxon rank-sum test. Categorical data were presented as percentages and compared by the $\chi^{2}$ test or the Fisher exact test when there were less than 5 values in a given cell. All statistical analyses were performed using SPSS statistical software (version 18.0, SPSS, Chicago, Illinois).

\section{Results}

A total of 365 patients with STEMI planning for primary PCI were screened for eligibility, of whom 192 patients were not eligible for the following reasons: previous myocardial infarction (36 patients), previous coronary artery bypass grafting (CABG) or PCI (61 patients), renal failure (19 patients), cardiogenic shock (35 patients), pacemakers existing (8 patients), severe infectious (6 patients), malignant tumor (11 patients) and refusal (16 patients). A total of 173 patients with STEMI were randomized (1:1) to receive either TMZ or placebo prior to primary PCl. A total of 148 patients who finally received CMR were eligible for the final analysis (Figure 2).

\subsection{Patient characteristics}

The mean age of the total study cohort was $57 \pm 12$ years, and males accounted for $90 \%$. The administrations of statins, $\beta$-blockers, and ACEI/ARB had no significant difference between the two groups. There was no significant difference in time from symptom to balloon and other baseline 
characteristics between the TMZ and control group (Table 1). TIMI grade before reperfusion wasn't significant difference between the two groups. Thrombectomy occurred in 25 patients $(28.7 \%)$ in the TMZ group and 23 patients (26.7\%) in the control group. Compared with the control group, the proportion of patients of TIMI 3 grade after primary PCI was a little higher in TMZ group without significance $(94.3 \%$ vs. $88.4 \% ; p=0.308)$, which was shown in Table 2.

\subsection{Primary endpoint}

Infarction size, MVO, AAR and MSI were measured by CMR, and there were two experienced CMR readers validated the results. The intra- and inter-observer variability on infarction size were $0.01 \pm 0.03$ and $0.02 \pm 0.05$, respectively (Figure 1 ). Compared with the control group, patients with STEMI who were administered TMZ prior to primary $\mathrm{PCl}$ had a reduction in infarction size percentage (expressed as a percentage of the LV mass, $22 \pm 12 \%$ [ $n=74$ ] vs. $27 \pm 13 \%[n=74] ; p=0.011$ ), absolute infarction mass $(28 \pm 18 g$ [n=74] vs. $35 \pm 19 g$ [n=74]; $p=0.022)$ and lower proportion of MVO $(29.7 \%$ [22/73] vs. $52.7 \%$ [39/74]; $p=0.007)$, meanwhile had an increase in MSI ( $48 \pm 20 \%$ vs. $39 \pm 27 \% ; p=0.008)$, which was showed in Table 3. The plasma levels of high sensitive troponin T (hsTnT) at $12 \mathrm{~h}(\mathrm{p}=0.028)$ and $24 \mathrm{~h}(\mathrm{p}=0.034)$ after reperfusion in TMZ group were significantly reduced than control group, which suggested TMZ reduced reperfusion injury in primary PCl (Figure 3, Table 4).

\subsection{Major adverse cardiovascular events}

Compared with control group, fewer occurrences of cumulative MACEs were observed in TMZ group without significance (Figure 4). During the one-year follow-up, the incidence of readmission for acute heart failure was lower in the TMZ group than control group $(8.0 \%$ vs. $14.0 \%, p=0.234)$. A total of 9 patients died during the follow-up, 4 in TMZ group and 5 in control group. There was no significant difference in the incidence of recurrent myocardial infarction or all-cause death (Figure 4, Table 5).

\section{Discussion}

TMZ is a clinically effective anti-ischemic agent, which reduces fatty acid oxidation while stimulates glucose oxidation by inhibiting long-chain 3-ketoacyl CoA thiolase. It has been reported that TMZ appeared to improve clinical outcomes in patients with AMI by significantly reducing all-cause mortality and other MACEs $[3,12]$. Our trial has demonstrated improved outcomes: TMZ treatment initiated prior to primary $\mathrm{PCl}$ could reduce infarction size and MVO, and make improvement of MSI in patients with STEMI. Besides, CMR was firstly used to evaluate the effect of TMZ on infarction size and MSI in patients with STEMI in our trial. Other trials have proven that TMZ therapy makes improvement of LVEF, and reduce reperfusion damage for patients with STEMI, especially for non-thrombotic patients [8, 13-16], and TMZ treatment commenced prior to $\mathrm{PCl}$ also made improvements in left ventricular end-diastolic volume and decrease in brain natriuretic peptide (BNP) level in patients with AMI [17], all of which were in line with the results of our clinical trial. 
Previous animal experiments suggested TMZ effectively reduced infarction size in animal models $[18,19]$. Early treatment of TMZ, especially in the acute ischemia-reperfusion phase, could reduce reperfusion myocardial injury through various mechanisms: TMZ modulates the substrate metabolism by shifting fatty acid oxidation to glucose oxidation during reperfusion $[2,4,5]$. TMZ could reduce the intracellular acidosis and deposition of intracellular sodium and calcium, prevent the membrane damage caused by the oxygen free radicals, and reduce white blood cells [20-22]. TMZ pretreatment could significantly inhibit myocardial apoptosis, and its cardiac protective effect appeared to be mediated by the blockade of the mitochondrial apoptotic pathway [23]. TMZ treatment significantly activates AMPK and ERK signaling pathway, and inhibits MMP-2 and MMP-9 expression, which leads to a reduction of oxidative stress in ischemia-reperfusion hearts $[5,21]$. All of the above mechanisms may contribute to reducing the infarction size for STEMI

Some previous researches show no significant improvement of $T M Z$ on the prognosis of angina pectoris after recent successful PCI [24]. However, a meta-analysis has reported that early TMZ therapy had overall benefits upon total MACEs in patients with AMI [12]. Our study provides additional evidence on the protective effect of the TMZ on relieving reperfusion injury in patients with STEMI after PCI. These inconsistent findings may be due to the following reason: The benefits efficacy of TMZ may depend on the timing and dosage of administration, and may also depend on patient status, and TMZ is likely to be more effective in the acute myocardial ischemia phase. Some previous studies did not give an adequate dosage of TMZ before the reperfusion, which might underestimate the cardioprotective effect of TMZ [13]. Our study treated STEMI patients with a loading dose of $60 \mathrm{mg} T \mathrm{TMZ}$ before PCl followed by TMZ for a total of 12 months to benefit these patients. It has been reported that pre-procedural oral TMZ administration significantly reduced $\mathrm{PCl}$-induced myocardial injury in $\mathrm{PCl}$ for stable angina pectoris [25]. Our study has validated that TMZ reduces PCl-induced myocardial injury in primary PCl, and provides a reference for drug administration's dose and duration.

Infarct size is a major determinant of post STEMI mortality, so limiting the extent of infarct size in STEMI is a major therapeutic target [26]. MSI also predicts the outcome of STEMI after reperfusion, and has important implications for prognosis [9]. It is our hypothesis that TMZ reduces infarct size and improve MSI by ameliorating reperfusion injury. Here, we show that an early therapy of TMZ could significantly reduce infarct size, and increase MSI simply by being administered before reperfusion. TMZ did not reduce mortality in our study which might be due to the insufficient sample size, and further evidence is needed to assess potential longer-term clinical benefits in a larger clinical trial.

\subsection{Study limitation}

There were some limitations in this study. Firstly, not all the CMR exams were performed exactly on the 7th day after $\mathrm{PCl}$ in our study, which might affect the final results of the observed variables due to the rapidly changing pathophysiological processes of the cardiac tissue [27]. Secondly, we used CMR data at 7 days post-STEMI as the endpoint. Although scar myocardial size performed at 1 month from the acute episode should be a more reliable measurement, but previous research has taken the infarction size, MVO, 
AAR and MSI measured with one week as a good alternative [28]. Furthermore, $14 \%$ of patients enrolled did not undergo the CMR for primary end-point for different reasons, and this attrition rate was as we projected and is similar to those in other STEMI trials using MRI [29]. Finally, this study was a singlecenter study, and our conclusion needed further validation in large-scale randomized studies.

\section{Conclusions}

This randomized study showed that $\mathrm{TMZ}$ initiated prior to primary $\mathrm{PCl}$, reduced myocardial infarct size and MVO, and improved MSI significantly, possibly by reducing reperfusion injury, making it a promising treatment for evaluation in larger randomized studies.

\section{Declarations}

This work was supported by grants from the China Cardiovascular Association (2018-CCA-CMVD-04), the Beijing Special fund for Development (SF2020-2-5012) and natural science foundation project (81870178).

Declarations: All authors have reported that they have no relationships relevant to the contents of this paper to disclose. All authors declare that they have no conflict of interest.

Availability of data: Contact the corresponding author (qiangeng9396@263.net) to get relevant data.

Authors' contributions $\varangle$ "QG, JZC, JXS and AX analyzed and interpreted the STEMI patients' data. LT analyzed CMR data, DW, GJ, CYD enrolled patients, ZY performed the CMR examination, and QG was a major contributor in writing the manuscript. All authors read and approved the final manuscript

Ethics approval: The study complied with the Declaration of Helsinki. Informed consent was obtained from all individual participants included in the study. The trial was registered on ClinicalTrials.gov (registration number: NCT02826616).There is no potential conflicts of interest. The study was approved by the Beijing Ethics Association and the Ethics Committee of the Chinese PLA General Hospital

\section{References}

1. Kallistratos MS, Poulimenos LE, Giannitsi S, Tsinivizov P, Manolis AJ. Trimetazidine in the Prevention of Tissue Ischemic Conditions. Angiology. 2019;70:291-8.

2. Marzilli M, Vinereanu D, Lopaschuk G, Chen Y, Dalal JJ, Danchin N, et al. Trimetazidine in cardiovascular medicine. Int J Cardiol. 2019;293:39-44.

3. Kim JS, Kim CH, Chun KJ, Kim JH, Park YH, Kim J, et al. Effects of trimetazidine in patients with acute myocardial infarction: data from the Korean Acute Myocardial Infarction Registry. Clin Res Cardiol. 2013;102:915-22.

4. Ma N, Bai J, Zhang W, Luo H, Zhang X, Liu D, et al. Trimetazidine protects against cardiac ischemia/reperfusion injury via effects on cardiac miRNA21 expression, Akt and the Bcl2/Bax 
pathway. Mol Med Rep. 2016;14:4216-22.

5. Liu Z, Chen JM, Huang H, Kuznicki M, Zheng S, Sun W, et al. The protective effect of trimetazidine on myocardial ischemia/reperfusion injury through activating AMPK and ERK signaling pathway. Metabolism. 2016;65:122-30.

6. Wu S, Chang G, Gao L, Jiang D, Wang L, Li G, et al. Trimetazidine protects against myocardial ischemia/reperfusion injury by inhibiting excessive autophagy. J Mol Med (Berl). 2018;96:791-806.

7. Zhong Y, Zhong P, He S, Zhang Y, Tang L, Ling Y, et al. Trimetazidine Protects Cardiomyocytes Against Hypoxia/Reoxygenation Injury by Promoting AMP-activated Protein Kinase-dependent Autophagic Flux. J Cardiovasc Pharmacol. 2017;69:389-97.

8. Di Pasquale P, Lo Verso P, Bucca V, Cannizzaro S, Scalzo S, Maringhini G, et al. Effects of trimetazidine administration before thrombolysis in patients with anterior myocardial infarction: short-term and long-term results. Cardiovasc Drugs Ther. 1999;13:423-8.

9. Eitel I, Desch S, Fuernau G, Hildebrand L, Gutberlet M, Schuler G, et al. Prognostic significance and determinants of myocardial salvage assessed by cardiovascular magnetic resonance in acute reperfused myocardial infarction. J Am Coll Cardiol. 2010;55:2470-9.

10. Lonborg J, Vejlstrup N, Kelbaek H, Botker HE, Kim WY, Mathiasen AB, et al. Exenatide reduces reperfusion injury in patients with ST-segment elevation myocardial infarction. Eur Heart J. 2012;33:1491-9.

11. Sousa-Uva Franz-JosefNMiguel, Ahlsson A, Alfonso F, Adrian P, Banning U, Benedetto, et al. 2018 ESC/EACTS Guidelines on myocardial revascularization. Eur Heart J. 2019 Jan 7;40(2):87-165.

12. Li Y, Wang D, Hu C, Zhang P, Zhang D, Qin S. Efficacy and Safety of Adjunctive Trimetazidine Therapy for Acute Myocardial Infarction: A Systematic Review and Meta-Analysis. Cardiology. 2016;135:18895.

13. Effect of 48-h. intravenous trimetazidine on short- and long-term outcomes of patients with acute myocardial infarction, with and without thrombolytic therapy; A double-blind, placebo-controlled, randomized trial. The EMIP-FR Group. European Myocardial Infarction Project-Free Radicals. Eur Heart J. 2000;21:1537-46.

14. Li R, Tang X, Jing Q, Wang Q, Yang M, Han X, et al. The effect of trimetazidine treatment in patients with type 2 diabetes undergoing percutaneous coronary intervention for AMI. Am J Emerg Med. 2017;35:1657-61.

15. Shehata M. Cardioprotective Effects of Oral Trimetazidine in Diabetic Patients With Anterior Wall Myocardial Infarction Treated with Thrombolysis. Cardiol Res. 2014;5:58-67.

16. Soukoulis V, Boden WE, Smith SC Jr, O'Gara PT. Nonantithrombotic medical options in acute coronary syndromes: old agents and new lines on the horizon. Circ Res. 2014;114:1944-58.

17. Demirelli S, Karakelleoglu S, Gundogdu F, Tas MH, Kaya A, Duman H, et al. The Impact of Trimetazidine Treatment on Left Ventricular Functions and Plasma Brain Natriuretic Peptide Levels in Patients with Non-ST Segment Elevation Myocardial Infarction Undergoing Percutaneous Coronary Intervention. Korean Circ J. 2013;43:462-7. 
18. Noble MI, Belcher PR, Drake-Holland AJ. Limitation of infarct size by trimetazidine in the rabbit. Am J Cardiol. 1995;76:41B-4B.

19. Senturk T, Cavun S, Avci B, Yermezler A, Serdar Z, Savci V. Effective inhibition of cardiomyocyte apoptosis through the combination of trimetazidine and $\mathrm{N}$-acetylcysteine in a rat model of myocardial ischemia and reperfusion injury. Atherosclerosis. 2014;237:760-6.

20. Tritto I, Wang P, Kuppusamy P, Giraldez R, Zweier JL, Ambrosio G. The anti-anginal drug trimetazidine reduces neutrophil-mediated cardiac reperfusion injury. J Cardiovasc Pharmacol. 2005;46:89-98.

21. Gong W, Ma Y, Li A, Shi H, Nie S. Trimetazidine suppresses oxidative stress, inhibits MMP-2 and MMP-9 expression, and prevents cardiac rupture in mice with myocardial infarction. Cardiovasc Ther. 2018;36:e12460.

22. Zheng S, Du Y, Peng Q, Fan X, Li J, Chen M. Trimetazidine Protects Against Atherosclerosis by Changing Energy Charge and Oxidative Stress. Med Sci Monit. 2018;24:8459-68.

23. Liu YC, Li L, Su Q, Liu T, Tang ZL. Trimetazidine pretreatment inhibits myocardial apoptosis and improves cardiac function in a Swine model of coronary microembolization. Cardiology. 2015;130:130-6.

24. Ferrari R, Ford I, Fox K, Challeton JP, Correges A, Tendera M, et al. Efficacy and safety of trimetazidine after percutaneous coronary intervention (ATPCI): a randomised, double-blind, placebo-controlled trial. Lancet. 2020;396:830-8.

25. Bonello L, Sbragia P, Amabile N, Com O, Pierre SV, Levy S, et al. Protective effect of an acute oral loading dose of trimetazidine on myocardial injury following percutaneous coronary intervention. Heart. 2007;93:703-7.

26. Gregg W, Stone, Harry P, Selker H, Thiele MR, Patel JE, Udelson EM, Ohman,et al. Relationship Between Infarct Size and Outcomes Following Primary PCl: Patient-Level Analysis From 10 Randomized Trials. J Am Coll Cardiol. 2016 Apr 12;67(14):1674-83.

27. Ibanez B, Aletras AH, Arai AE, Arheden H, Bax J, Berry C, et al. Cardiac MRI Endpoints in Myocardial Infarction Experimental and Clinical Trials: JACC Scientific Expert Panel. J Am Coll Cardiol. $2019 ; 74: 238-56$.

28. Klug G, Mayr A, Schenk S, et al. Prognostic value at 5 years of microvascular obstruction after acute myocardial infarction assessed by cardiovascular magnetic resonance. Journal of cardiovascular magnetic resonance: official journal of the Society for Cardiovascular Magnetic Resonance. 2012;14(1):46.

29. Stone GW, Maehara A, Witzenbichler B, Godlewski J, Parise H, Dambrink JH, et al. Intracoronary abciximab and aspiration thrombectomy in patients with large anterior myocardial infarction: the INFUSE-AMI randomized trial. JAMA. 2012;307:1817-26.

\section{Tables}

Table 1 Baseline characteristics of trimetazidine group and control group 


\begin{tabular}{|c|c|c|c|}
\hline & \multicolumn{2}{|c|}{ Trimetazidine group ( $\mathrm{N}=87)$ Control group $(\mathrm{N}=86)$} & \multirow{2}{*}{$P$ value } \\
\hline Demographic characteristics & & & \\
\hline Male (\%) & $79(90.8)$ & $77(89.5)$ & 0.804 \\
\hline Age (years) & $57 \pm 11$ & $58 \pm 12$ & 0.638 \\
\hline \multicolumn{4}{|l|}{ Medical history } \\
\hline Hypertension (\%) & $41(47.1)$ & $51(59.3)$ & 0.128 \\
\hline Diabetes mellitus (\%) & $17(19.5)$ & $21(24.4)$ & 0.467 \\
\hline Hypercholesterolemia (\%) & $16(18.4)$ & $20(23.2)$ & 0.459 \\
\hline Smoker (\%) & $50(57.5)$ & $50(58.1)$ & 1.000 \\
\hline Premature CAD family (\%) & $14(16.1)$ & $13(15.1)$ & 1.000 \\
\hline Stroke (\%) & $4(4.6)$ & $6(7.0)$ & 0.535 \\
\hline Prior PCI & $3(3.4)$ & $6(7.0)$ & 0.329 \\
\hline Prior CABG & $1(1.1)$ & $1(1.2)$ & 1.000 \\
\hline \multicolumn{4}{|l|}{ Clinical manifestation at presentation } \\
\hline Weight (kg) & $74 \pm 12$ & $75 \pm 13$ & 0.439 \\
\hline $\mathrm{SBP}(\mathrm{mmHg})$ & $122 \pm 22$ & $122 \pm 23$ & 0.866 \\
\hline $\mathrm{DBP}(\mathrm{mmHg})$ & $71 \pm 13$ & $72 \pm 15$ & 0.679 \\
\hline Heart rate $(\mathrm{bpm})$ & $79 \pm 14$ & $80 \pm 15$ & 0.594 \\
\hline Symptom to balloon time (hour) & $5.8 \pm 2.6$ & $5.8 \pm 2.8$ & 0.927 \\
\hline Killip class 3 or $4(\%)$ & $2(2.3)$ & $3(3.5)$ & 0.682 \\
\hline Hemoglobin (g/L) & $145 \pm 17$ & $147 \pm 17$ & 0.346 \\
\hline White blood cell $\left(10^{9} / \mathrm{L}\right)$ & $11.14 \pm 3.56$ & $10.84 \pm 3.88$ & 0.598 \\
\hline Platelet count $\left(10^{12} / \mathrm{L}\right)$ & $223 \pm 76$ & $221 \pm 62$ & 0.806 \\
\hline Random glucose (mmol/l) & $7.54 \pm 2.50$ & $7.55 \pm 2.47$ & 0.995 \\
\hline Alanine aminotransferase & $40 \pm 30$ & $37 \pm 28$ & 0.519 \\
\hline LDL-c (mmol/l) & $2.90 \pm 0.94$ & $2.94 \pm 0.92$ & 0.772 \\
\hline Serum creatinine & $79 \pm 34$ & $77 \pm 18$ & 0.589 \\
\hline Blood urea nitrogen & $5.78 \pm 2.33$ & $5.57 \pm 1.48$ & 0.493 \\
\hline Baseline Brain Natriuretic Peptide & $(74,645)$ & $(73,439)$ & 0.299 \# \\
\hline \multicolumn{4}{|l|}{ Treatment after primary PCI } \\
\hline Aspirin and ticagrelor & $87(100.0)$ & $86(100.0)$ & 1.000 \\
\hline Statin & $87(100.0)$ & $86(100.0)$ & 1.000 \\
\hline Beta-blockers & 77 (88.5) & $78(90.7)$ & 0.804 \\
\hline ACEI/ARB & $58(66.7)$ & $54(62.8)$ & 0.635 \\
\hline
\end{tabular}

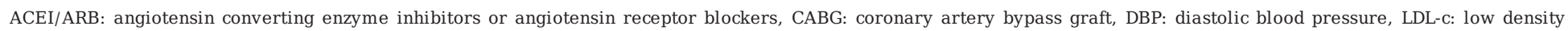
lipoprotein cholesterol, PCI: percutaneous coronary intervention, SBP: systolic blood pressure, \# non-parameter tests

Table 2 Angiographical and procedural characteristics of trimetazidine group and control group 


\begin{tabular}{lccc}
\hline & Trimetazidine group (N = 87) & Control group (N = 86) & $P$ value \\
\hline Pre TIMI grade flow, n (\%) & $66(75.9)$ & $72(83.7)$ & 0.548 \\
0 & $2(2.3)$ & $1(1.2)$ & \\
1 & $7(8.0)$ & $7(8.1)$ & \\
2 & $11(12.6)$ & $6(7.0)$ & \\
3 & & & 0.308 \\
Post TIMI grade flow, n (\%) & $1(1.1)$ & $1(1.2)$ & \\
0 & $1(1.1)$ & $4(4.7)$ & \\
1 & $3(3.4)$ & $5(5.8)$ & \\
2 & $82(94.3)$ & $76(88.4)$ & \\
3 & & & \\
Culprit Vessel, $\mathbf{n}$ (\%) & $1(1.1)$ & $0(0)$ & 0.497 \\
LM & $39(44.8)$ & $37(43.0)$ & 0.879 \\
LAD & $7(8.0)$ & $13(15.1)$ & 0.161 \\
LCX & $40(46.0)$ & $35(40.7)$ & 0.540 \\
RCA & $25(28.7)$ & $23(26.7)$ & 0.865 \\
Thrombectomy, n (\%) & $1.3 \pm 0.6$ & $1.3 \pm 0.7$ & 0.822 \\
Number. of stent per patients, n (\%) & $4(4.6)$ & $4(4.7)$ & 1.000 \\
IABP, n (\%) & &
\end{tabular}

IABP: intra aortic balloon pumping, TIMI: thrombolysis in myocardial infarction.

Table 3 Troponin $\mathrm{T}$ in trimetazidine group and control group after reperfusion

\begin{tabular}{lcccc}
\hline & Trimetazidine group (N =87) Control group (N =86) & $\begin{array}{c}\text { Difference } \\
\text { (95\% CI) }\end{array}$ & P value \\
\hline is troponin T, ng/l & & & & \\
) h & $700(2390)$ & $685(2580)$ & $26(-563$ to 616$)$ & 0.763 \\
i h & $3561(4250)$ & $4025(5580)$ & $-880(-1960$ to 200$)$ & 0.156 \\
$2 \mathrm{~h}$ & $3754(5410)$ & $5028(4070)$ & $-1469(-2923$ to -145$)$ & 0.028 \\
in & $3290(3550)$ & $4083(4420)$ & $-1458(-2719$ to -197$)$ & 0.034 \\
t8h & $2450(3060)$ & $2880(3170)$ & $-815(-1900$ to 269$)$ & 0.160 \\
\hline
\end{tabular}

Table 4 Primary endpoints as measured by cardiac magnetic resonance

\begin{tabular}{lccc}
\hline & Trimetazidine group (N =74) & Control group (N = 74) & $P$ value \\
\hline st ventricle end-diastolic volume, ml & $132 \pm 37$ & $139 \pm 40$ & 0.260 \\
farct size, Proportion of LV mass, \% & $22 \pm 12$ & $27 \pm 13$ & 0.011 \\
farct size, g & $28 \pm 18$ & $35 \pm 19$ & 0.022 \\
sea at risk, g & $57 \pm 31$ & $58 \pm 27$ & 0.853 \\
yocardial salvage index, \% & $48 \pm 20$ & $39 \pm 27$ & 0.008 \\
cidence of microvascular obstruction, (\%) & $22 / 74(29.7)$ & $39 / 74(52.7)$ & 0.007 \\
icrovascular obstruction, g & $0.8 \pm 1.8$ & $2.0 \pm 4.0$ & 0.017 \\
\hline
\end{tabular}


Table 5 Main adverse cardiac events for one year follow-up

Trimetazidine group $(\mathrm{N}=87)$ Control group $(\mathrm{N}=86) \quad P$ value

\begin{tabular}{lccc}
\hline ladverse cardiovascular events & $13(14.9)$ & $18(20.9)$ & 0.328 \\
roke, n (\%) & $1(1.1)$ & $0(0)$ & 1.000 \\
эcurrent myocardial infarction, n (\%) & $1(1.1)$ & $1(1.2)$ & 1.000 \\
əhospitalization for heart failure, n (\%) & $7(8.0)$ & $12(14.0)$ & 0.234 \\
l cause Death, n (\%) & $4(4.6)$ & $5(5.8)$ & 0.747 \\
\hline
\end{tabular}

\section{Figures}
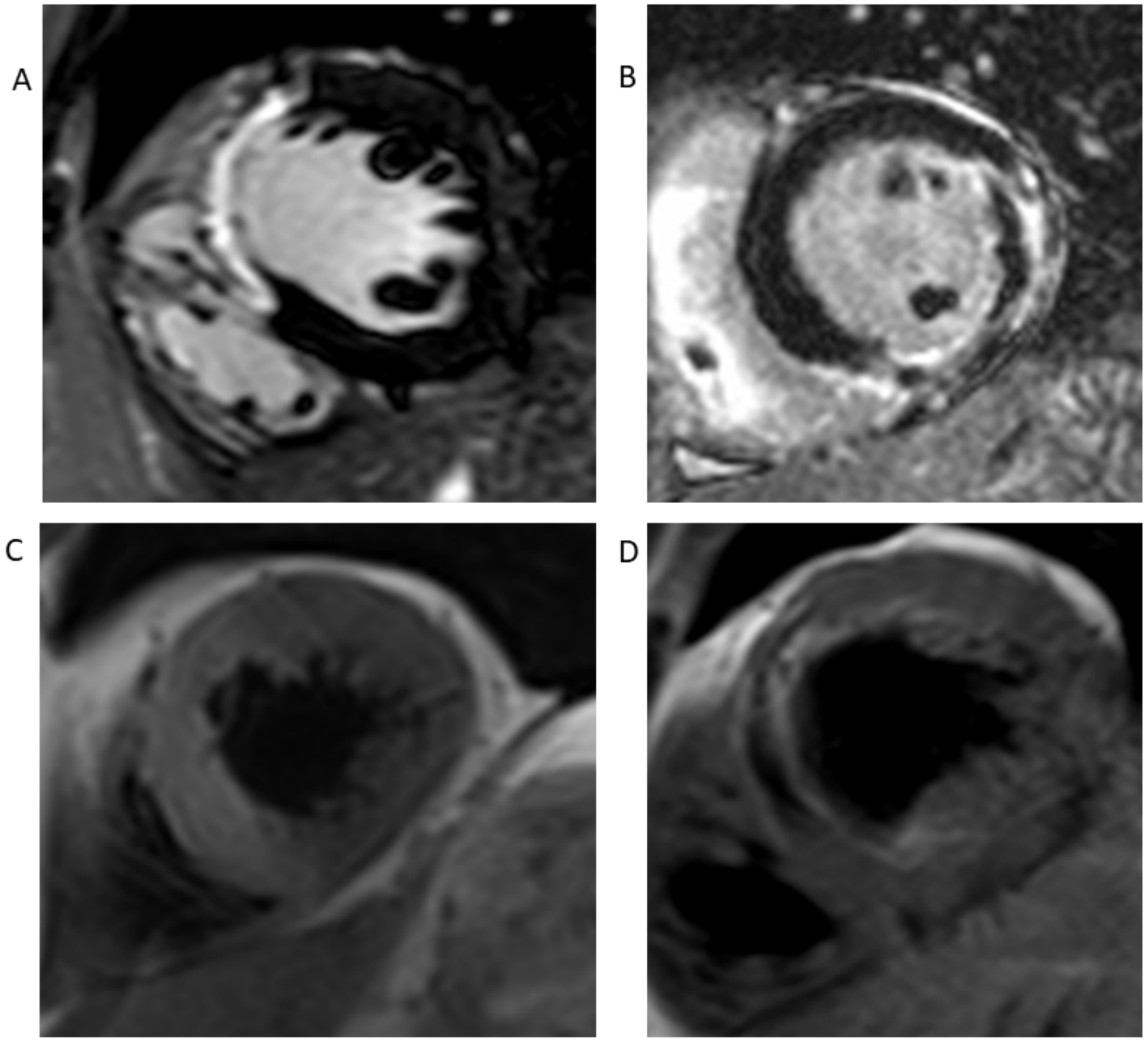
Figure 1

Examples of delayed enhancement and T2-weighted cardiac magnetic resonance images of patients with ST-segment elevation myocardial infarction (STEMI) which showed a lesion in the left anterior descending artery. Delayed enhancement image used for final infarction size analysis (A), and T2weighted image used for area at risk analysis (C, D), late gadolinium enhancement to assess myocardial infarction size and microvascular obstruction . T2 maps depict the area of myocardial edema.

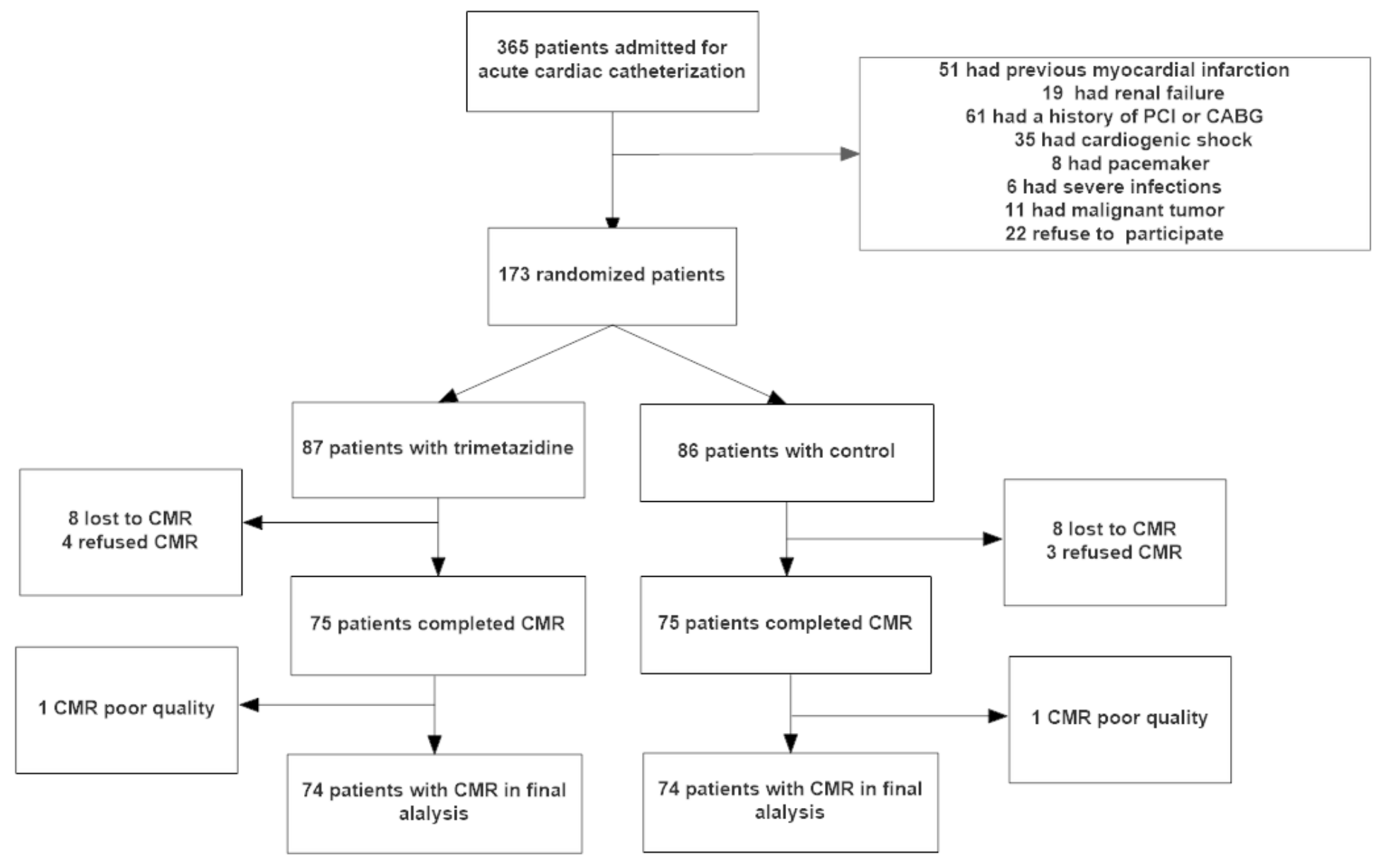

Figure 2

Flow chart of the study: A total of 173 patients were enrolled 
Dynamic changes of high sensitive Troponin $\mathrm{T}$ in reperfusion treatment

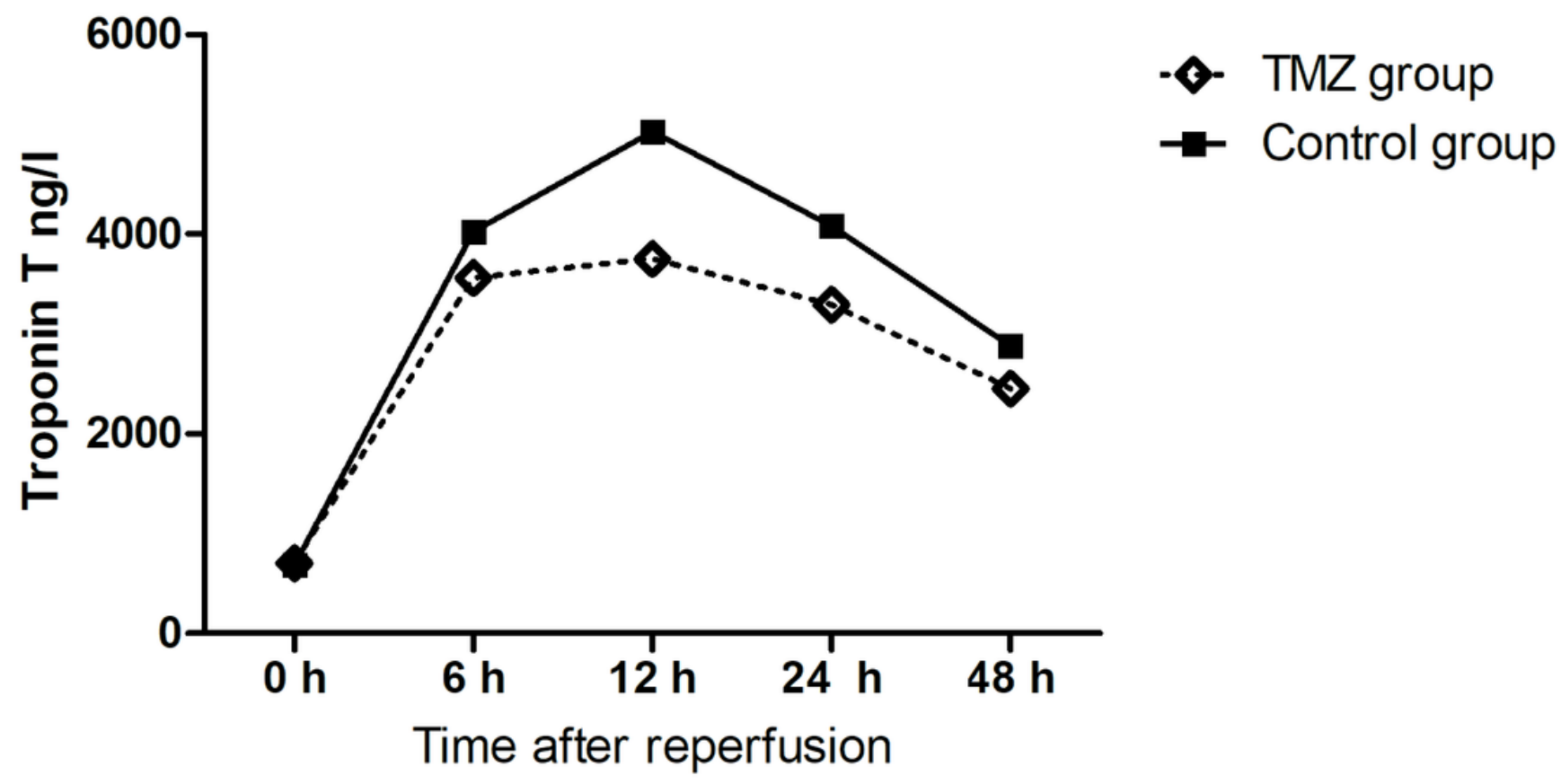

Figure 3

Dynamic changes of high sensitive Troponin T (hsTnT) in reperfusion treatment: The hsTnT level of patients in trimetazidine (TMZ) group (solid line) was significantly lower at $12 \mathrm{~h}$ and $24 \mathrm{~h}$ after reperfusion when compared to control ones (dashed line). 


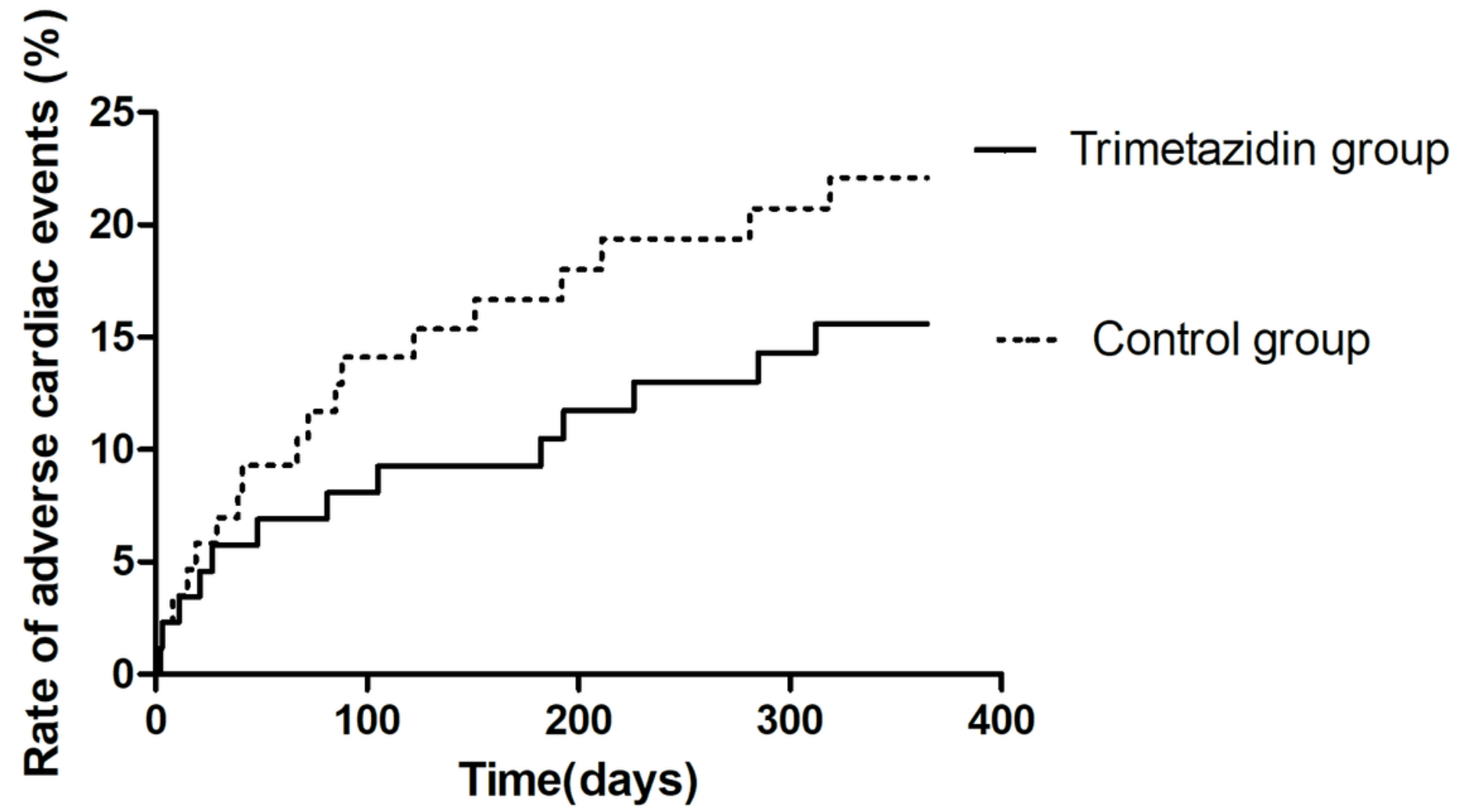

Figure 4

Main adverse cardiac events in the study patients: Less occurrences of cumulative major adverse events were observed in trimetazidine (TMZ) group compared with the control group. 